Fowler, W. A.: 1964, Rev. Mod. Phys. 36, 545.

Friedman, J. L. and Schutz, B. F.: 1975, Astrophys. J. 199, L157.

Hulse, R. A.: 1975, Astrophys. J. 195, L51.

Hulse, R. A. and Taylor, J. H.: 1975, Astrophys. J. 195, L51.

Kraft, R. P.: 1975, in H. Gursky and R. Ruffini (eds.), Neutron Stars, Black Holes and Binary X-Ray Sources,

D. Reidel Publishing Company, Dordrecht, Holland, p. 235.

Masters, A. R. and Roberts, D. H.: 1975, Astrophys. J. 195, L107.

McCluskey, G. E. and Kondo, Y.: 1971, Astrophys. Space Sci. 10, 464.

Meier, D. L., Epstein, R. I., Arnett, W. D., and Schramm, D. N.: 1976, Astrophys. J. 204, 869.

Middleditch, J. and Nelson, J.: 1976, Astrophys. J. 208, 567.

Misner, C. W., Thorne, K. S., and Wheeler, J. A.: 1973, Gravitation, W. H. Freeman and Company, San Francisco.

Ostriker, J. P. and Gunn, J. E.: 1969, Astrophys. J. 157, 1395.

Primini, F., Rappaport, S., Joss, P. C., Clark, G. W., Lewin, W., Li, F., Mayer, W., and McClintock, J.: 1976, Astrophys. J. 210, L71.

Rappaport, S., Joss, P. C., and McClintock, J. E.: 1976, Astrophys. J. 206, L103.

Roberts, D. H., Masters, A. R., and Arnett, W. D.: 1976, Astrophys. J. 203, 196.

Ross, D. K.: 1975, J. Phys. A: Math. Gen. 8, 1398.

Smarr, L. L. and Blandford, R.: 1976, Astrophys. J. 207, 574.

Sofia, S.: 1967, Astrophys. J. 148, L 1.

Sofia, S.: 1971, Nature 234, 155.

Sutantyo, W.: 1974, Astron. Astrophys. 34, 339.

Tananbaum, H. and Tucker, W. H.:1974, in R. Giacconi and H. Gursky (eds.), X-Ray Astronomy, D. Reidel

Publishing Company, Dordrecht, Holland, p. 207.

van den Heuvel, E. P. J. and de Loore, C.: 1973, Astron. Astrophys. 25, 387.

van Horn, H. M., Sofia, S., Savedoff, M. P., Duthie, J. G., and Berg, R. A.: 1975, Science 188, 930.

Wheeler, J. C. and Hansen, C. V.: 1971, Astrophys. Space Sci. 11, 373.

Wheeler, J. C., Lecar, M., and McKee, C. F.: 1975, Astrophys. J. 200, 145.

Wilson, J. R.: 1971, Astrophys. J. 163, 209.

\title{
Addendum
}

Dr W. Sutantyo kindly pointed out to us an inadvertent error in the article by McCluskey and Kondo (1971) referenced in the current paper. The Equation (6) in that paper should read

$$
\frac{a}{a_{0}}=\frac{A+B}{2 A+B-C-1} .
$$

As a result, the Tables Ib and Ic also change slightly; the corrected Tables Ib and Ic are given. These corrections do not alter our conclusions in that article. In fact, as may be seen, the corrected results are slightly more in favor of the binary not breaking up after the supernova explosion. 


\section{TABLE Ib}

Computed values of $a / a_{0}$ and $e$ for $C=0.20$

(Upper entry is $a / a_{0}$; lower entry is $e$ )

\begin{tabular}{lllllll}
\hline$B / A$ & 0.10 & 0.20 & 0.40 & 0.60 & 0.80 & 0.95 \\
\hline 0.20 & - & - & - & 4.00 & 1.67 & 1.28 \\
& - & - & - & 0.79 & 0.53 & 0.43 \\
0.60 & - & - & 5.00 & 2.00 & 1.40 & 1.19 \\
& - & - & 0.82 & 0.58 & 0.43 & 0.37 \\
1.00 & - & 6.00 & 2.33 & 1.60 & 1.29 & 1.15 \\
& - & 0.85 & 0.62 & 0.47 & 0.37 & 0.33 \\
2.00 & 2.10 & 1.83 & 1.50 & 1.30 & 1.17 & 1.09 \\
& 0.57 & 0.51 & 0.41 & 0.34 & 0.29 & 0.26 \\
5.00 & 1.28 & 1.24 & 1.17 & 1.12 & 1.07 & 1.04 \\
& 0.28 & 0.26 & 0.23 & 0.21 & 0.19 & 0.18 \\
\hline
\end{tabular}

TABLE Ic

Computed values of $a / a_{0}$ and $e$ for $C=0.50$

(Upper entry is $a / a_{0}$; lower entry is $e$ )

\begin{tabular}{lllllll}
\hline $3 . / A$ & 0.10 & 0.20 & 0.40 & 0.60 & 0.80 & 0.95 \\
\hline 0.20 & - & - & - & - & 3.33 & 1.92 \\
& - & - & - & - & 0.80 & 0.67 \\
0.60 & - & - & - & 4.00 & 2.00 & 1.55 \\
& - & - & - & 0.82 & 0.65 & 0.58 \\
1.00 & - & - & 4.67 & 2.29 & 1.64 & 1.39 \\
& - & - & 0.83 & 0.67 & 0.57 & 0.51 \\
2.00 & 3.00 & 2.44 & 1.85 & 1.53 & 1.33 & 1.23 \\
& 0.72 & 0.66 & 0.57 & 0.50 & 0.44 & 0.42 \\
5.00 & 1.38 & 1.33 & 1.26 & 1.19 & 1.14 & 1.10 \\
& 0.38 & 0.37 & 0.34 & 0.32 & 0.30 & 0.29 \\
\hline
\end{tabular}

